\title{
Relajación del Piso Pélvico Suspensión con Cinta de Dacrón*
}

\author{
Dres.: Jaime Garcia M., Gabriel Uribe E., \\ Gabriel Tobón L. **
}

\section{Introducción}

Siguiendo el plan trazado en nuestro Servicio de Ginecología desde 1967 de tratar el prolapso uterino en la mujer joven utilizando la técnica de la suspensión con dacrón, queremos presentar ahora 12 casos más, intervenidas en los últimos años.

Este trabajo es la continuación de otro ya presentado en la Revista Colom. biana de Obstetricia y Ginecología Vol. XXIII No. 6 de 1972 (1) por los docto= res Baquero y Tuberquia en donde se informó de 33 pacientes tratadas con este método. En aquel entonces se justificó la intervención en mujeres jóvenes que deseaban conservar su capacidad de reproducción o en aquellas otras a quienes una histerectomía podria representar un problema de rechazo por parte del esposo.

Desgraciadamente la selección de los casos es cada vez más dificil y reducida debido a que las causas principales de relajación del piso pélvico en estas mujeres, trauma del parto mal conducido y la multiparidad, van desapareciendo poco a poco en nuestro medio debido a la mayor y mejor atención

Estudio de 12 pacientes intervenidas en el Hospital Universitario San Vicente de Paúl de Medellín de Enero de 1974 a Junio de 1979.

* Profesores Departamento de Ginecología y Obstetricia - Facultad de Medicina - Universidad de Antioquia-Medellin - 1979. médica que se brinda a la comunidas y a la educación que se imparte en relación con campañas de planificación familiar.

Evidentemente en todas las revisiones ginecológicas se encuentran muchas pacientes con grados moderados o leves de relajación del piso pélvico que no necesitan ningún tipo de tratamiento y que si se seleccionaran para tratamiento quirúrgico elevarían grandemente el número de casos; pero como lo anotamos antes, sólo justificamos la técnica para aquellas pacientes jóvenes con histeroceles grados 11 y III.

\section{Técnica quirúrgica}

Seguimos practicando la técnica descrita por Durfee en 1966 (3), que básicamente consiste en lo siguiente:

1. Laparotomía infraumbilical tipo Pfannenstiel hasta cavidad peritoneal. Fig. 1 (4).

2. Identificación de los ligamentos redondos y su salida del abdomen a través del anillo inguinal; a través de este último se pasa una pinza de Pean que abre un túnel entre las dos hojas del ligamento ancho, inmediatamente por debajo y a lo largo del ligamento redondo, hasta llegar a la cara posterior del útero a nivel del orificio cervical interno. Fig. 2. 


\section{Figura 1}

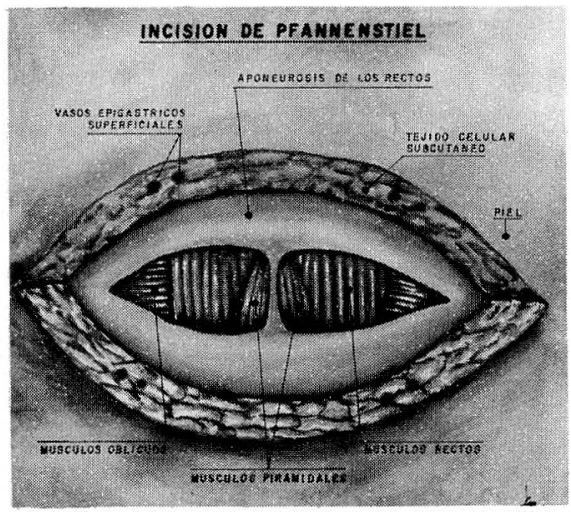

3. Apertura de una pequeña ventana en el peritoneo de la cara posterior del útero a nivel del istmo. Fig. 2

4. La punta de la pinza de Pean se saca por esta ventana y toma uno de los extremos de una cintilla de dacrón de unos $12 \mathrm{~cm}$ de longitud por $1 \mathrm{~cm}$ de ancho. Se extrae la pinza y con ella la parte de la cintilla de dacrón que se había tomado. Figs. 3 y 4 .

5. Se repite la maniobra en el lado opuesto: Se saca la punta de la pinza por la ventana practicada en la pared posterior del útero, se toma el otro extremo de la cintilla de dacrón y se saca fuera de cavidad abdominal por el túnel practicado entre las hojas del ligamento ancho inmediatamente por debajo del ligamento redondo.

6. Fijación de la cintilla de dacrón a la cara posterior del útero con puntos separados de hilo de algodón o de dacrón No. 000 y cierre de la ventana peritoneal utilizando catgut simple No. 000. Figs. 5 y 6 .

7. Fijación de los extremos de la cintilla de dacrón a la cara interna de la aponeurosis del oblicuo menor y a la cara interna de la aponeurosis de los músculos rectos del abdomen en los ángulos de la herida quirúrgica, utilizan- do puntos separados de hilo de dacrón o de algodón. Fig. 7.

8. Antes de la fijación anterior se debe ajustar la tensión de la cintilla de manera que el útero no se eleve en forma excesiva, pero tampoco dejarla tan floja que no se produzca ninguna corrección del prolapso.

9. Cierre de la pared abdominal por planos y de acuerdo con las técnicas habituales.

10. Practicar reconstrucción perinea! cuando el caso lo permita.

\section{Objetivos de la técnica}

Se debe obtener un restablecimiento adecuado de la posición de los órganos pélvicos y de las estructuras de sostén, utilizando material de sutura permanente y no reactivo en forma tal que se conserve la función de reproducción.

\section{Figura 2}

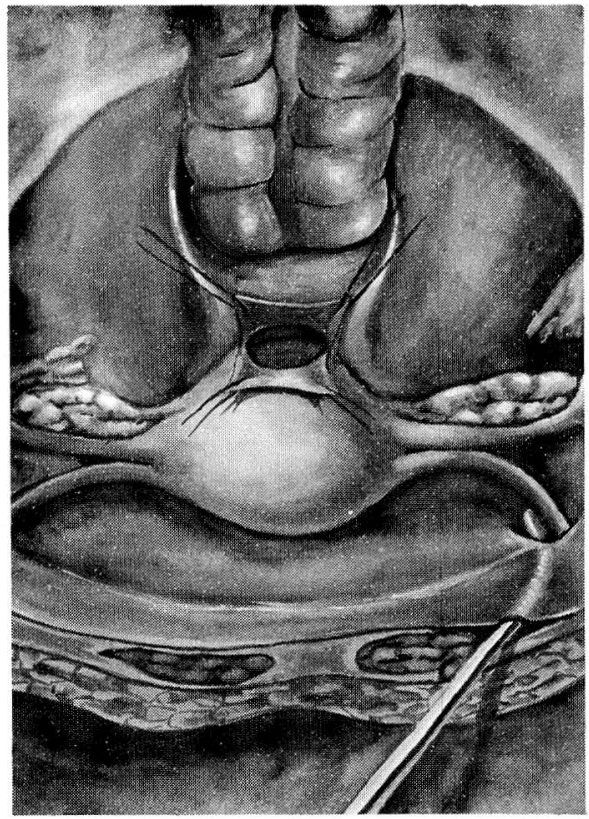


Figura 3

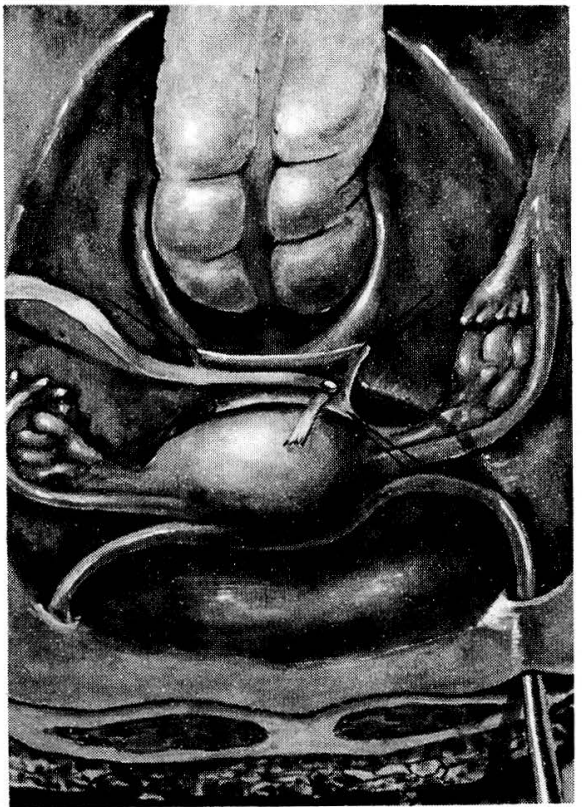

Durante muchos años se utilizó con estos mismos objetivos la técnica de la intervención de Manchester - Fothergill a la que se le encontraron los siguientes inconvenientes $(1,4)$ :

a) Las recidivas del prolapso son muy frecuentes pues la intervención no corrige las posiciones posteriores del útero.

b) La incidencia de esterilidad es alta vical por la alteración del factor cer-

c) En los pocos casos en que se logra el embarazo la frecuencia del aborto es alta debido a la incompetencia cervical que presentan muchas de esas pacientes.

\section{Objetivos generales}

Se analiza la técnica quirúrgica y se estudia la evolución de las pacientes: resultado de la intervención, recidivas del prolapso y sus posibles causas, embarazos y la forma en que terminaron.

\section{Material y métodos}

El material de este trabajo lo constituyen 12 pacientes que fueron tratadas con la técnica descrita. La intervención le fue practicada a mujeres jóvenes con prolapso integral II y III que deseaban tener más hijos. El control de las pacientes ha ido de 3 meses a 5 años.

\section{CUADRO 1}

\section{RELAJACION DEL PISO PELVICO SUSPENSION CON CINTILLA DE DACRON $1973-1979$}

\begin{tabular}{|c|c|c|}
\hline Edad & & No. Pacientes \\
\hline $15-19$ & & 1 \\
\hline $20-24$ & & 3 \\
\hline $25-29$ & & 5 \\
\hline $30-34$ & $=$ & 3 \\
\hline & Total & 12 \\
\hline
\end{tabular}

Cuadro 1. Edad de las pacientes: Sólo una de las pacientes tenía 35 años de edad, el resto de ellas tenían menos de 30 años. La paciente más joven, de 17 años, inició su prolapso a la edad de 15 años después de un parto laborioso intervenido con fórceps.

\section{CUADRO 2}

\begin{tabular}{|c|c|}
\hline Paridad & No. Pacientes \\
\hline 1 & 6 \\
\hline 2 & 4 \\
\hline 3 & 2 \\
\hline Total & 12 \\
\hline
\end{tabular}

Cuadro 2. Paridad. El cuadro muestra cómo la mayoría de las pacientes tienen muy baja paridad. Es raro encontrar pacientes jóvenes con más de tres hijos que quieren someterse a este tipo de tratamiento. No hemos tenido pacientes nulíparas con prolapso. 
Figura 4

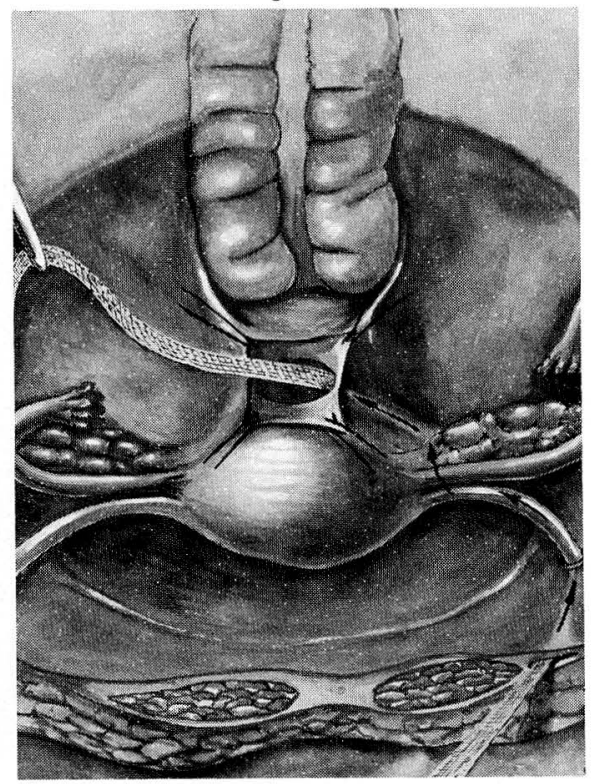

CUADRO 3

\begin{tabular}{|c|c|}
\hline Clasificación del Prolapso & No. Pacientes \\
\hline 1 & 0 \\
\hline 11 & 1 \\
\hline 111 & 11 \\
\hline Total & 12 \\
\hline
\end{tabular}

Cuadro 3. Hallazgos pélvicos: 11 de las pacientes presentaron un prolapso integral III y solamente una presentó prolapso II. No se tuvieron en cuenta para el tratamiento pacientes con grados menores de prolapso, pues consideramos que no son tributarias de tratamiento quirúrgıco.

En todos los casos el prolapso se desarrolló más o menos rápidamente después de su último o único parto. El estado general de las pacientes y sus exámenes de laboratorio mostraron cómo el estado nutricional no desempeñó el menor papel en la etiología.
CUADRO 4

\begin{tabular}{lc}
\hline Intervención practicada & No. Pacientes \\
\hline $\begin{array}{l}\text { Suspensión con Dacrón } \\
\text { Suspensión con Dacrón y } \\
\text { plastia posterior }\end{array}$ & 7 \\
$\begin{array}{l}\text { Suspensión con Dacrón y } \\
\text { Salpingolisis }\end{array}$ & 1 \\
$\begin{array}{l}\text { Suspensión con Dacrón y } \\
\text { Resección en cuña de ovarios }\end{array}$ & 1 \\
\hline \multicolumn{1}{c}{ Total } & 12 \\
\hline
\end{tabular}

Cuadro 4. En 7 pacientes la única intervención practicada fue la corrección del prolapso por medio de la suspensión con dacrón. En 3 pacientes se practicó además una reconstrucción perineal posterior. A otra se le complementó el tratamiento con salpingolisis de la trompa izquierda y a una quinta se le practicó resección en cuña de ambos ovarios por poliquistosis ovárica. Ninguna de las pacientes presentó complicaciones post-operatorias o intrahospitalarias y fueron dadas de alta al tercero o cuarto dia, para continuar su control en forma ambulatoria.

\section{Figura 5}

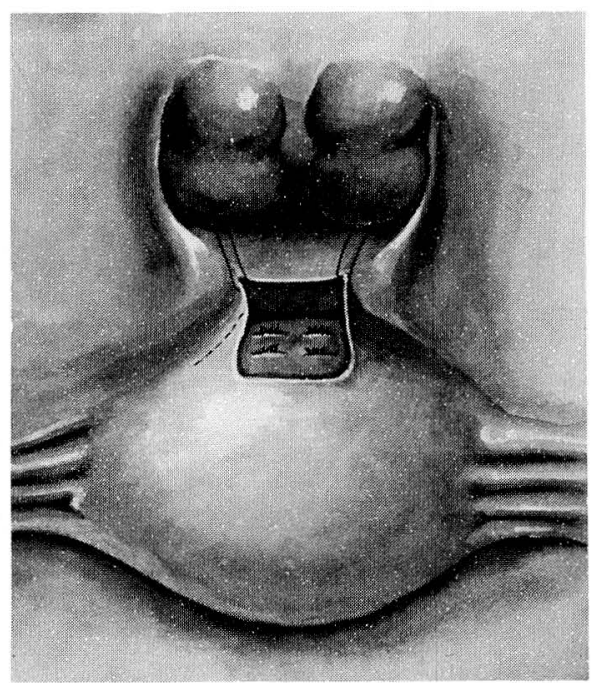


CUADRO 5

\begin{tabular}{lc}
\hline Resultados & No. Pacientes \\
\hline Corrección del Prolapso & 11 \\
Recidiva del Prolapso & 1 \\
\hline \multicolumn{1}{c}{ Total } & 12 \\
\hline
\end{tabular}

Cuadro 5. Resultados: Una de las pacientes presentó recidiva del prolapso el cual fue corregido 2 años más tarde con la misma técnica y con buenos resultados. Otra de las pacientes a quien se le practicó plastia posterior se infectó fuera del servicio y no consultó oportunamente; el resultado fue la recidiva de su desgarro perineal.

\section{CUADRO 6}

No. de pacientes con Emba- Método Evolución razo posterior a la interven- del parto Posterior ción.

2 Cesárea Normal

Cuadro 6. Dos de estas pacientes se embarazaron posteriormente y sus partos fueron atendidos por cesárea.

\section{Figura 6}

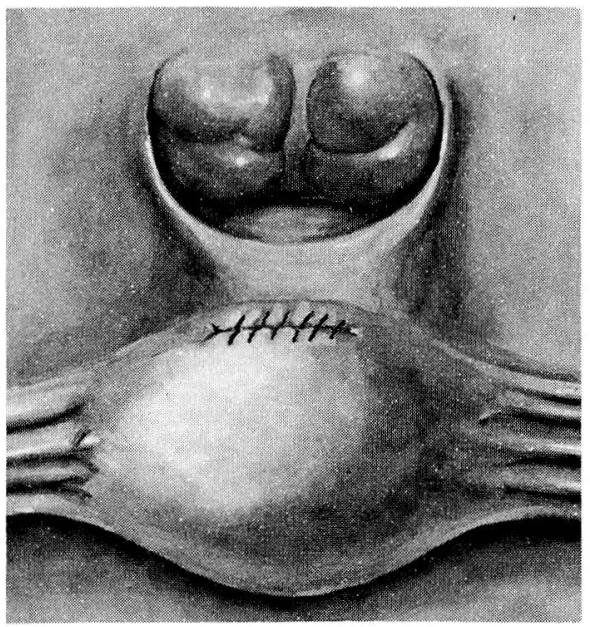

Figura 7

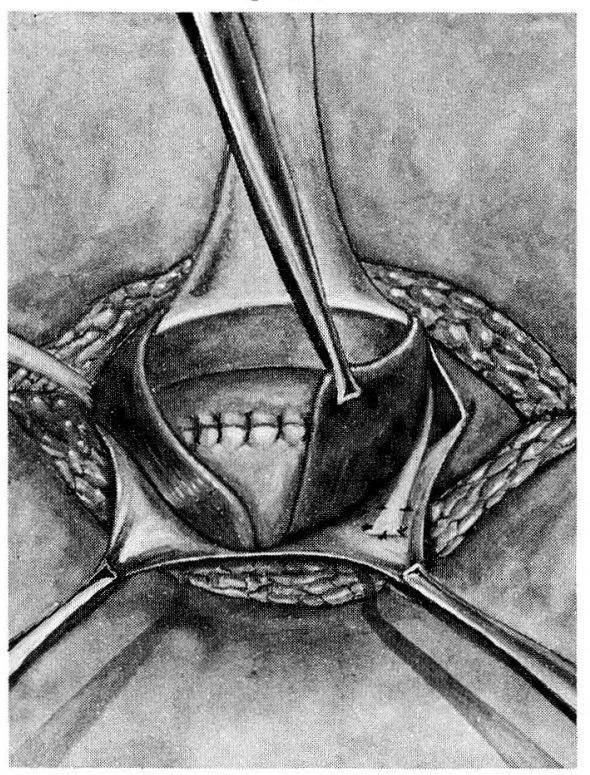

\section{Conclusiones}

La relajación del piso pélvico en mujeres plantea un reto para el ginecólogo quien debe resolver qué técnica quirúrgica va a realizar para corregir esta patologia y garantizarle a la paciente la conservación de la función reproductiva. Consideramos que la técnica de la suspensión con dacrón es la más adecuada pues en todas las pacientes se consiguió el objetivo propuesto.

Estamos convencidos que el parto debe ser atendido por cesárea electiva, sin trabajo de parto, pues aunque la cintilla no impide la dilatación cervical, el parto por vía vaginal sí puede provocar recidiva del prolapso.

Se les ha sugerido a las pacientes la planificación en los primeros años, además es importante advertirles la clase de intervención efectuada para que esté en condiciones de informar posteriormente, en caso de posible histerectomía, la clase de cirugía practicada. 
En el trabajo presentado por los doctores Baquero y Tuberquia (1) se decía que era el primero con esta técnica descrito en el país y que la bibliografía era demasiado escasa. Siete años más tarde al presentar este otro trabajo nos hemos encontrado con que a la bibliografía de entonces no hay nada que agregar ni dentro ni fuera del país.

\section{Resumen}

Se presenta la revisión de 12 pacientes a quienes se les practicó la técnica de la suspensión con dacrón para tratar la relajación del piso pélvico en mujeres jóvenes. Se describe la técnica quirúrgiç. se analizan los resultados y se dan algunas recomendaciones.

\section{Bibliografía}

1 BAQUeRO B. y TUBerquia C.: Prolapso uterino en mujeres jóvenes tratadas con suspensión con dacrón. Revista Colombiana de Obstetricia y Ginecología Vol. XXIII No. 6. pág. 547, 1972.

2 BERNAL B.: Histeropexia en mujeres jóvenes. Ginecología y Obstetricia de Méjico. Vol. 33 No. 197, 1973.

3 DURFEE R. B.: Operaciones de suspensión para tratar prolapso de órganos genitales. Clínicas Obstétricas y Ginecológicas, diciembre de 1966, pág. 1047.

4 TELINDE R. W.: Ginecología Operatoria. Editorial Bernades, Buenos Aires. Cuarta Edición, págs. 134 y 5C6, 1971. 